\title{
$\gamma$-hadron correlation measurements to study jets fragmentation with ALICE at LHC
}

\author{
Yaxian Mao $^{1, \mathrm{a}}$, Gustavo Conesa Balbastre ${ }^{2}$, Masato Sano ${ }^{3}$, Yves Schutz ${ }^{4,5}$, Daicui Zhou ${ }^{1}$ \\ ${ }^{1}$ Institute of Particle Physics, Central China Normal University, Wuhan, China \\ ${ }^{2}$ Laboratori Nazionali di Frascati, INFN, Frascati, Italy \\ ${ }^{3}$ Graduate School of Pure and Applied Sciences, University of Tsukuba, Tsukuba, Japan \\ ${ }^{4}$ IN2P3, Nantes, France \\ ${ }^{5}$ CERN, Genève, Suisse
}

Received: 11 November 2007 / Revised: 15 August 2008 / Published online: 26 September 2008

(C) Springer-Verlag / Società Italiana di Fisica 2008

\begin{abstract}
The possibility to study the fragmentation function of jets with energies below $50 \mathrm{GeV}$, which cannot be reconstructed in the heavy-ion environment, is explored. We propose to measure the imbalance distribution between prompt photon and charged hadrons with the ALICE experiment at LHC. In the present article, the case of protonproton collisions at $\sqrt{s}=14 \mathrm{TeV}$ is examined with MonteCarlo simulations. Performances for prompt photon identification and rejection of the $\pi^{0}$ decay photons are presented. We find that the measurement in proton-proton is meaningful with respect to statistical and systematic errors for photon energies larger than $20 \mathrm{GeV}$.
\end{abstract}

\section{Introduction}

The objective of the heavy-ion physics program at ultrarelativistic energies is to establish the properties of strongly interacting matter at high energy densities. The experimental program pursued at RHIC with proton-proton $(p p)$ and nucleus-nucleus $(A A)$ collisions from $\sqrt{s_{N N}}=60$ to $200 \mathrm{GeV}$ has demonstrated [1], through a variety of observables, that the properties of the matter formed in heavyion collisions at these ultra-relativistic energies can be interpreted in terms of partonic degrees of freedom. However, the matter formed appears to be strongly interacting rather than behaving as a free gas as predicted at asymptotic temperatures by non-perturbative Lattice Quantum Chromo Dynamics (LQCD) calculations [4]. To establish this picture, the measurement of high transverse momentum $\left(p_{\mathrm{T}}\right)$ particles $\left(p_{\mathrm{T}} \gg T\right.$, where $T$ is the temperature of the medium, of the order of several hundreds of $\mathrm{MeV} / c$ ) has played a central role. Such measurements indicated that the production

a e-mail: maoyx@iopp.ccnu.edu.cn of high $p_{\mathrm{T}}$ hadrons is quenched in central $A A$ collisions [2]. This observation has been interpreted by the energy loss experienced by hard scattered partons traversing a color dense medium. Such an observable is however not well adapted to extract quantitative data on the properties of the medium formed in $A A$ collisions, due to its weak sensitivity on the medium density, mainly because of trigger biases [3].

Energy densities within reach at the CERN Large Hadron Collider (LHC) with heavy-ion collisions at centre of mass energies of several TeV's will exceed several times the critical value at which LQCD calculations predict the transition from hadronic matter to deconfined partonic matter. This unexplored energy regime creates much more favourable opportunities to observe the partonic phase exploiting the abundantly produced hard probes.

In particular, the more sensitive observable provided through the measurement of the re-distribution in phasespace of hadrons from the jet fragmentation when the hard scattered parton traverses the medium [5] will be within reach. Such a re-distribution is best seen by measuring the Fragmentation Function (FF), i.e., the distribution of charged hadrons in a jet as a function of their fractional momentum, $z=p_{\mathrm{T}_{\mathrm{h}}} / E_{\mathrm{T}}^{\mathrm{jet}}$, with respect to the jet energy, $E_{\mathrm{T}}^{\mathrm{jet}}$, and by comparing the vacuum FF (measured in $p p$ collisions or peripheral $A A$ collisions) and the medium modified $\mathrm{FF}$ (measured in central $A A$ collisions). The difficulty, however, is to measure $z$, since the jet energy, in the so called cone algorithm, must be calculated ideally by adding the contributions from all hadrons falling in a cone with radius $R=\sqrt{\Delta \eta^{2}+\Delta \phi^{2}}=1$ around the jet direction in $(\eta, \phi)$. Such a measurement is not feasible in $A A$ collisions for low jet energies because of the large background generated by the soft underlying events limiting the jet reconstruction to energies above $50 \mathrm{GeV}[6]$. 
The limiting threshold can however be lowered by tagging jets with prompt photons in the jet energy range where standard jet finding algorithms cannot be invoked. In a recent study [7], it was demonstrated that such a measurement is feasible with the detectors of the ALICE experiment [8], based on the estimation of statistical and systematic errors anticipated during one standard year of data taking. Although the energy range within reach is limited by the counting rate of jets with energy of the order of $20 \mathrm{GeV}$ (because of the low acceptance for photon measurements in ALICE), it was concluded that medium properties can be measured if the effects on the FF are of at least $5 \%$.

The photon tagging measurement still requires to identify the jet of hadrons emitted opposite to the photon direction, which remains challenging in the $A A$ environment. This is not the case if one measures prompt-photon-hadron correlations. The method [9-11] rests on the fact that under given kinematical conditions, the imbalance parameter $z_{\gamma-\mathrm{h}}=-\vec{p}_{\mathrm{T}_{\mathrm{h}}} \cdot \vec{p}_{\mathrm{T}_{\gamma}} /\left|p_{\mathrm{T}_{\gamma}}\right|^{2}$ is in a Leading Order (LO) kinematics equivalent to the fragmentation variable $z$. The kinematical conditions which verify this equivalence are the following:

- The hadrons must be produced from the fragmentation of a hard scattered parton, i.e., in $A A$ to exclude hadrons from the soft underlying event, $p_{\mathrm{T}_{\mathrm{h}}}>p_{\mathrm{T}_{\mathrm{h}}}^{\text {cut }} \gg \Lambda_{\mathrm{QCD}}, T$.

- The photon must be produced directly from a hard partonic process, i.e., $p_{\mathrm{T}_{\gamma}}>p_{\mathrm{T}_{\gamma}}^{\text {cut }} \gg \Lambda_{\mathrm{QCD}}, T$ and it must not be produced in a jet fragmentation.

- The range over which the equivalence is verified is given by $p_{\mathrm{T}_{\mathrm{h}}}^{\text {cut }} / p_{\mathrm{T}_{\gamma}}^{\text {cut }} \lesssim z_{\mathrm{h} \gamma} \lesssim 1$, therefore to probe the broadest range the photon and hadron momenta cuts must be very asymmetric, $p_{\mathrm{T}_{\gamma}}^{\text {cut }} \gg p_{\mathrm{T}_{\mathrm{h}}}^{\text {cut }}$.

Experimentally, the choice of the kinematics constraints must also take into account the counting rate within reach and the efficiency of isolation cut methods [7] to identify prompt photons. With ALICE, a reasonable choice is $p_{\mathrm{T}_{\gamma}}^{\text {cut }} \sim$ $20 \mathrm{GeV} / c$. The cut on the hadrons momentum is then optimally chosen to avoid the soft $p_{\mathrm{T}}$ region $\left(p_{\mathrm{T}} \lesssim 2 \mathrm{GeV} / c\right)$, where the FF is dominated in $A A$ collisions by the underlying event, i.e. $p_{\mathrm{T}_{\mathrm{h}}}^{\mathrm{cut}} \sim 2 \mathrm{GeV} / c$.

In the present article, we discuss the feasibility of measuring the $z_{\gamma-\mathrm{h}}$ distribution in $p p$ collisions at $\sqrt{s}=14 \mathrm{TeV}$ with the ALICE experimental setup at LHC with the perspective of applying this measurement to $A A$ collisions and extracting quantitative information on the properties of the medium formed under such conditions.

\section{Photons and charged hadrons detection in ALICE}

The ALICE experiment [8] has been designed to measure and identify a large fraction of the particles produced in $p p$ and $A A$ collisions within very high particle densities $\left(\mathrm{d} N /\left.\mathrm{d} \eta\right|_{\eta=0} \lesssim 8000\right)$. Charged particles are tracked in the central barrel detectors (ITS and TPC), covering the pseudorapidity domain $-0.9 \leq \eta \leq+0.9$ and the full azimuth. The physical track-finding efficiency (including geometrical acceptance and reconstruction efficiency) is above $85 \%$ over the whole $p_{\mathrm{T}}$ range in $p p$ as well as in $A A$. The transverse momentum resolution is $\Delta p_{\mathrm{T}} / p_{\mathrm{T}} \leq 5 \%$ for the high $p_{\mathrm{T}}$ particles of interest for our study. Although the tracking detectors have been optimised for particle identification, this possibility will not be used in our study.

Photons are detected and identified with the Photon Spectrometer (PHOS) [12]. It covers a limited acceptance at mid-rapidity: $-0.12 \leq \eta \leq+0.12$ and $\Delta \phi=100^{\circ}$. It is split along the azimuth in 5 modules of $\Delta \phi=20^{\circ}$, each highly segmented in $56 \times 64$ lead tungstate crystals of $2.2 \times 2.2 \times 18 \mathrm{~cm}^{3}$. Since it is foreseen, that for the LHC startup not all the modules will be installed, we have considered for the present study two modules only. In addition we have considered that the material in front of these two modules is equivalent to $10 \%$ of radiation length. Under these conditions, the measured energy resolution of PHOS is $\Delta E / E=3 \% / \sqrt{E}$ and its position resolution in the transverse directions $\Delta x(z) / x(z)=1-3 \mathrm{~mm} / \sqrt{E}$. A Charge Particle Veto (CPV) detector identifies charged particles impinging on PHOS.

A crucial aspect of the photon-hadron correlation measurement is the ability to identify prompt photons among all the photons generated in $p p$ and $A A$ collisions. The major sources of background are decay photons, mainly from $\pi^{0}$. In the $\pi^{0} p_{\mathrm{T}}$ domain of interest, above $20 \mathrm{GeV} / c$, the Lorenz boost contracts the relative angle between $\pi^{0}$ decay photons to small values $\left(\Delta \gamma_{1} \gamma_{2}<0.8^{\circ}\right.$ for a symmetric decay). The distance of PHOS from the interaction point being $460 \mathrm{~cm}$, the two showers developing in PHOS overlap and the 2 decay photons are reconstructed as one single photon. This configuration provides, however, the opportunity to discriminate photons from overlapped decay photons based on an analysis of the cluster shape: a cluster with axial symmetry in $(x, z)$ signs a photon, whereas an asymmetric cluster signs overlapped decay photons [7]. This method is not selective enough to reduce the flux of decay photons to a negligible low level. Isolation selections must be applied to obtain a sufficiently clean sample of prompt photons. The isolation criteria defines the level of hadronic activity in a cone axed on the direction of the prompt photon candidate. In $p p$ collisions, this selection rejects most of the remaining decay photons. Obviously, in $A A$ collisions because of the high multiplicity underlying events, the isolation is less efficient but remains an excellent means to identify efficiently prompt photons [7] in particular when the high $p_{\mathrm{T}} \pi^{0}$ production is quenched by the medium as observed at RHIC. The isolation selection has the additional advantage to reject as well 
fragmentation photons or bremsstrahlung photons present in $A A$ collisions. These two contributions alter the equivalence between imbalance distribution and FF [9-11].

\section{Monte-Carlo data production}

The elementary $2 \rightarrow 2$ hard processes at the origin of LO prompt photons are gluon Compton scattering $(g q \rightarrow \gamma q)$ and quark annihilation $(q \bar{q} \rightarrow \gamma g)$. We call them $\gamma$-jet events. Neutral pion mesons, the only source of decay photons considered in the present study, are produced through fragmentation of hard scattered partons in any $2 \rightarrow 2$ process. We call these events jet-jet events. Using the PYTHIA event generator [13], $p p$ events including either of the $\gamma$-jet or jet-jet process were generated at $\sqrt{s}=14 \mathrm{TeV}$. The PYTHIA version was 6.214 with the default parton distribution function CTEQ4L [14]. Initial and final state radiation were switched on and the intrinsic transverse momentum of the colliding partons was set to the default value $k_{\mathrm{T}}=1 \mathrm{GeV} / c$. Fragmentation photons are produced during the parton shower evolution, as final state radiation. Their contribution in PYTHIA was evaluated to about $58 \%$ of the direct photon produced at $20 \mathrm{GeV} / c$. Identified photons in PHOS from jet-jet events thus include mainly $\pi^{0}$ decay photons and fragmentation photons.

Since the momentum spectra of the final state particles follows a steeply falling power law, the events have been generated in bins of $p_{\mathrm{T}}^{\text {hard}}$, where $p_{\mathrm{T}}^{\text {hard }}$ refers to transverse momentum of the outgoing hard scattered partons. The number of events generated for each $p_{\mathrm{T}}^{\text {hard }}$ bin were normalised to the cross section calculated by PYTHIA. The events of interest for the present study were selected by triggering on prompt photons (in the case of $\gamma$-jet events) or on $\pi^{0}$ (in the case of jet-jet events) produced in the laboratory system inside an acceptance slightly larger than the geometrical acceptance of two PHOS modules $\left(\Delta \eta=0.26, \Delta \phi=42^{\circ}\right)$. Jet-jet events were triggered by $\pi^{0}$ with $p_{\mathrm{T}}$ larger than $1 \mathrm{GeV} / \mathrm{c}$ entering the acceptance of PHOS. This trigger considerably reduces the computing time needed to generate a statistically significant sample of photons detected in PHOS.

The generated particles from the accepted events were stored for later analysis and are referred to as the kinematics in the following. The generated particles were then tracked through the ALICE experimental setup using the GEANT3 [15] transport model (version v1-8). The hits produced during the transport were reconstructed with the standard ALICE reconstruction algorithms to produce reconstructed tracks for charged particles and reconstructed and identified photons. Finally, the analysis was performed on the reconstructed events stored in the Event Summary Data (ESD). All steps, from event generation to the final data analysis were performed with AliRoot [16] the ALICE framework for data generation and processing. The version used is v4-05-Release together with Root v5-15-08 [17].

The $\gamma$-jet events were generated in six bins of $p_{\mathrm{T}}^{\text {hard }}$ $(\mathrm{GeV} / c),[5,10],[10,20],[20,30],[30,40],[40,50],>50$ and with $10^{5}$ events per bin. The jet-jet events were generated in 24 bins, [5, 10], [10, 15], [15, 20], [20, 25], [25, 30], $[30,35],[35,40],[40,45],[45,50],[50,60],[60,70], \ldots$, $>200$, also with $10^{5}$ events per bin. More events have to be generated in the case of jet-jet events than in the case of $\gamma$-jet events to collect a statistically significant sample of events for $\pi^{0}$ production at a given $p_{\mathrm{T}}$ since fragmentation hadrons carry only a fraction of the jet energy distributed in $z$ following a piecewise exponentially decreasing function.

\section{Results}

The photon $p_{\mathrm{T}}$ spectra (Fig. 1) follow a power law distribution $p_{\mathrm{T}}^{-n}$ with $n=3.9 \pm 1.0$ for $\gamma$-jet photons in the $p_{\mathrm{T}}$ range from 10 to $90 \mathrm{GeV} / c$ and $n=5.1 \pm 0.6$ for jet-jet photons in the $p_{\mathrm{T}}$ range from 10 to $50 \mathrm{GeV} / c$. The resulting Monte Carlo counting rates are obviously much lower for the decay photon spectrum than for the prompt photon spectrum. This is a result, as mentioned earlier, of the fact that the $\pi^{0}$ and hence the decay photons sample only as a fraction of the jet energy from where they fragment. To reach a significantly larger sample of decay photons would require unaffordable computing resources.

The resulting ratio of the generated number of $\gamma$-jet photons to the number of jet-jet photons entering the PHOS acceptance (Fig. 2) is equal to 0.07 at $20 \mathrm{GeV} / c$.

Two different procedures to select prompt photons in PHOS were applied: the first one is based on the shape of the shower developing in PHOS $[18,19]$ and the second is an isolation cut [7]. Data have been further corrected to take into account the fact that the trigger particles were generated over a solid angle slightly larger than the PHOS acceptance. We find that the shower shape analysis, for distinguishing direct photons and decay photons merging into a single cluster, allows us to enrich substantially the data sample with direct photons. However the ratio $\gamma$-jet photon to jet-jet photon, of about 0.2 at $p_{\mathrm{T}}$ of $20 \mathrm{GeV} / c$ (Fig. 2), remains too low. This is due to the fact that at this energy the decay photons can still be separated in PHOS. Identifying the decay photons with an event-by-event invariant mass analysis is possible but has not been considered because the isolation analysis plays the same role.

The performances of the different methods to identify prompt photons applying isolation criteria have been extensively discussed [7]. A set of parameters defining the phase space in which the hadronic activity is calculated and thresholds on the hadronic activity have been deduced to optimise the isolated photon identification efficiency and minimise 


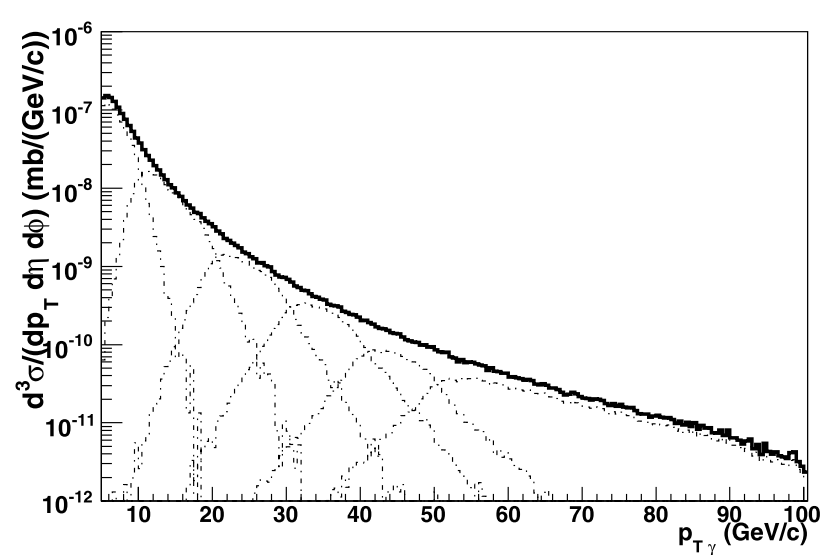

Fig. 1 Monte Carlo (PYTHIA) $\gamma$-jet photon spectrum (left) and jetjet photon spectrum $(r i g h t) p_{\mathrm{T}}$ spectra in $p p$ collisions at $\sqrt{s}=14 \mathrm{TeV}$. $\gamma$-jet photons are produced in $q g \rightarrow \gamma q$ and $q \bar{q} \rightarrow \gamma g$ hard processes and jet-jet photons in final state radiation during the parton shower

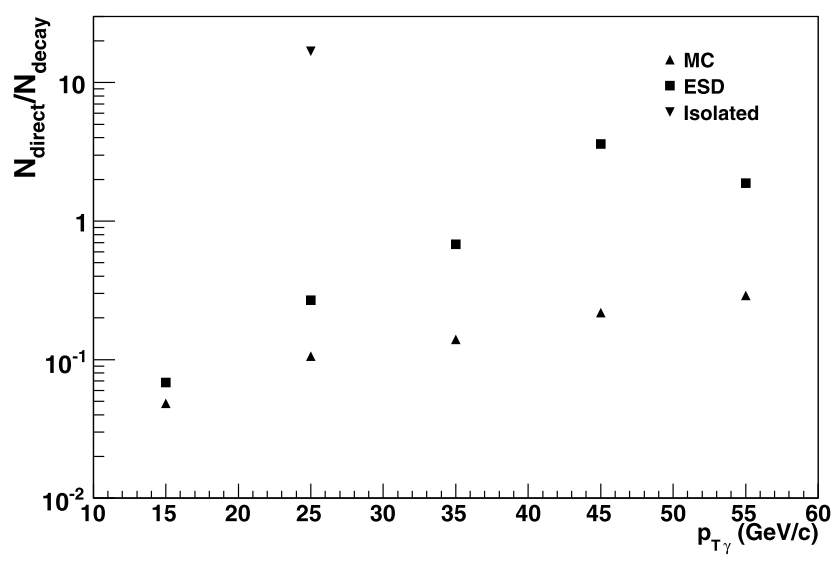

Fig. 2 Ratio of the $p_{\mathrm{T}}$ spectra of $\gamma$-jet photon and jet-jet photon in the PHOS acceptance $\left(\Delta \eta=0.26, \Delta \phi=42^{\circ}\right)$ in $p p$ collisions at $\sqrt{s}=14 \mathrm{TeV}$. Up triangle corresponds to the generated particles, rectangle to particles detected in 2 PHOS modules $(\Delta \eta=0.24$, $\Delta \phi=40^{\circ}$ ) and identified as photons, and the down triangle to particles identified as isolated photons (value averaged over the $p_{\mathrm{T}}$ range with usable counting rate)

the contamination level from decay photons. The same parameters have been adopted for the present studies although they have been originally established from generated data rather than fully reconstructed data. A cone of $R=0.3$ defines the spatial extent around the prompt photon candidate over which the hadronic activity is calculated as the sum of the $p_{\mathrm{T}}$ for all charged tracks and neutral clusters (Fig. 3). For $\gamma$-jet events, most of the hadronic activity stays below a few $\mathrm{GeV} / c$ due to the few hadrons from the underlying $p p$ events. The high $p_{\mathrm{T}}$ activity observed in the reconstructed events is interpreted as due to photons which have been converted before reaching PHOS, the CPV failing to identify the conversion electrons and the two electron tracks contributing to the hadronic activity. For jet-jet events the hadronic

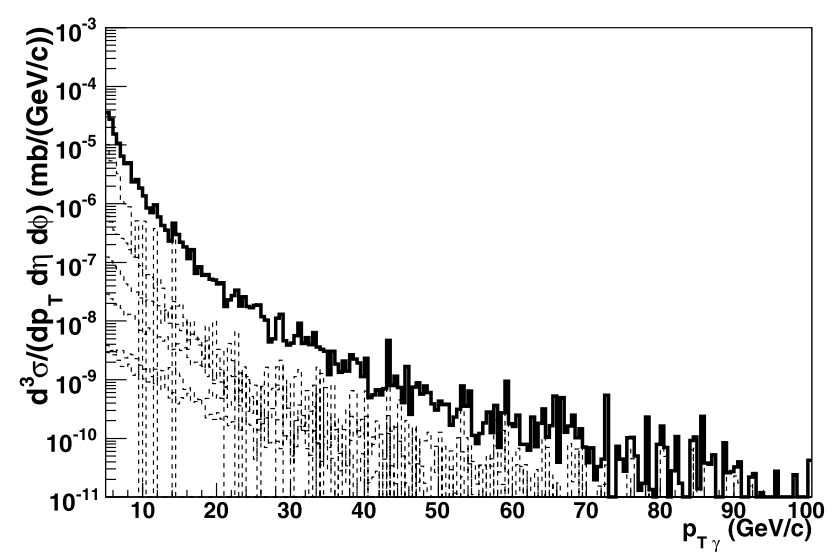

evolution or in the decay of neutral pions fragmenting jets from $2 \rightarrow 2$ hard processes. Simulation bins in $p_{\mathrm{T}}^{\text {hard }}$ normalised to the cross section are also displayed

activity is distributed over several tens of $\mathrm{GeV} / c$. Choosing the isolation cut parameter $\sum p_{\mathrm{T}}<2 \mathrm{GeV} / c$, the isolation efficiency to identify prompt photons is almost $100 \%$ over the entire $p_{\mathrm{T}}$ range and the efficiency to reject decay photons is larger than $90 \%$. After applying the two prompt photon selection procedures, the remaining contamination due to wrongly identified decay photons, averaged between 20 to $60 \mathrm{GeV} / c$, is as low as $0.5 \%$.

High energy photons enriched data samples will be collected thanks to a specialised PHOS trigger which efficiency for photons energies larger than $10 \mathrm{GeV}$ has been estimated to be $98 \%$ in $p p$ collisions. The identified prompt photon sample which can be accumulated with the two PHOS modules during the first year of LHC running $\left(\mathcal{L}=10^{30} \mathrm{~cm}^{-2} \mathrm{~s}^{-1}\right.$ and $\left.t=10^{7} \mathrm{~s}\right)$ in $p p$ collisions at $\sqrt{s}=14 \mathrm{TeV}$ (Fig. 4) will be sufficient to measure the prompt photon spectrum over a large $p_{\mathrm{T}}$ range between 20 and $80 \mathrm{GeV} / c$. Counting rates will limit the measurement, but not systematic errors which are dominated by the remaining decay photon and fragmentation photon background (the systematic errors have been arbitrarily multiplied by two to take into account additional source of photons in jet-jet events not considered in our simulation). Completing the originally foreseen PHOS setup with three additional modules will allow to improve the quality of the measurement (the counting rates scale with the number of modules), but additional material from tracking detectors put in front on some PHOS modules will limit this extension.

\section{Photon-charged hadron correlation}

In a previous study [20], based only on Monte Carlo generated particles modified by the parametrised detector re- 


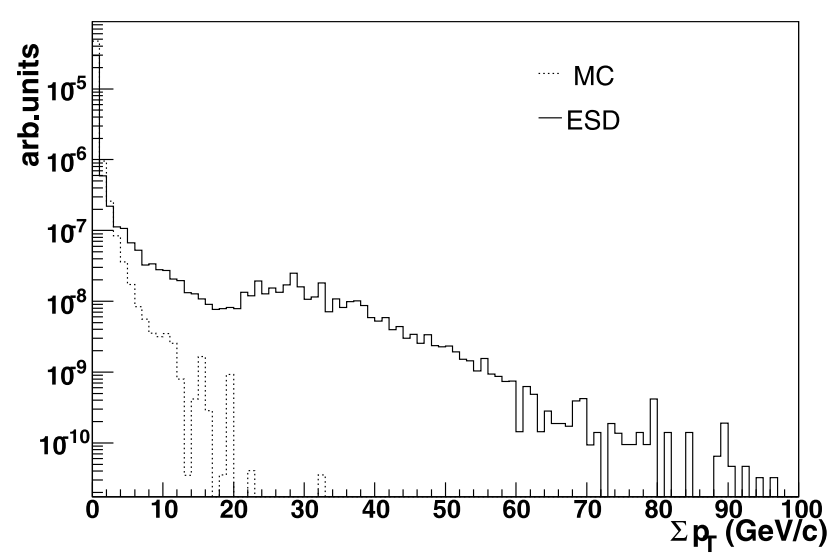

Fig. 3 Total transverse momentum of charged hadrons inside a cone with $R=0.3$ around a direct photon candidate calculated from Monte Carlo (PYTHIA generation) events (dashed line) and from events re-

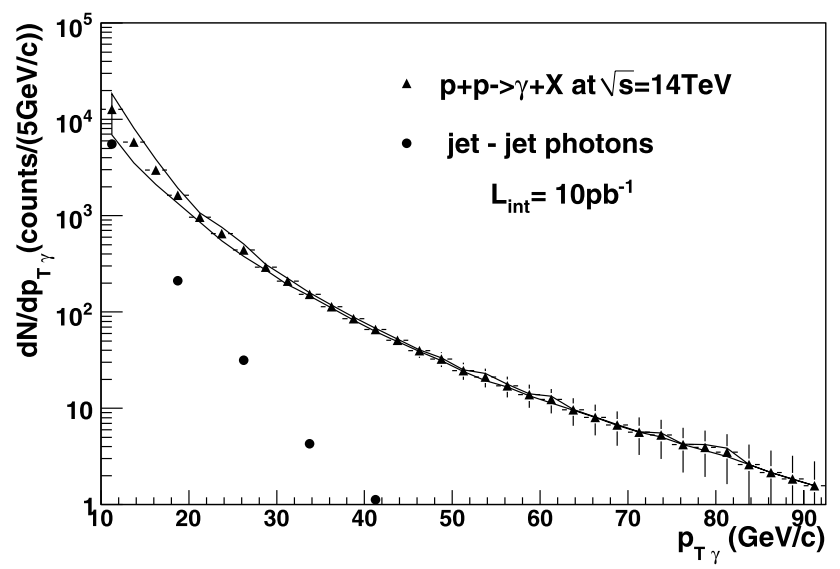

Fig. 4 Isolated photon counting rate that can be acquired with two PHOS modules during the first year of LHC running at nominal conditions in $p p$ collisions at $\sqrt{s}=14 \mathrm{TeV}$. The data points with error bars represents the total spectrum ( $\gamma$-jet plus jet-jet events) as it will be measured, the errors bars represent the statistical error of the measurement and the area around the data points represents the systematic errors due to contamination of imperfectly identified jet-jet photons (full circles)

sponse (no tracking was applied), prompt-photon-jet correlations have been studied. It was concluded that the FF tagged by photons can be measured with a statistically significant set of data for photons detected and identified in PHOS with energies of the order of $20 \mathrm{GeV}$. The FF can be mapped from $z=0.1$ up to $z=0.7$ in $p p$ collisions and up to $z=0.6$ in $P b P b$ collisions at $\sqrt{s_{N N}}=5.5 \mathrm{TeV}$, only limited by counting rate. It is estimated that modifications of the FF in this $z$ range can be measured at a $5 \%$ level.

The more simple observable suggested in Ref. [9-11] and which does not require to identify the jet opposite to the prompt photon was implemented as follows. First, prompt photons with energy above a given threshold $\left(p_{\mathrm{T}_{\gamma}}^{\text {cut }}=\right.$

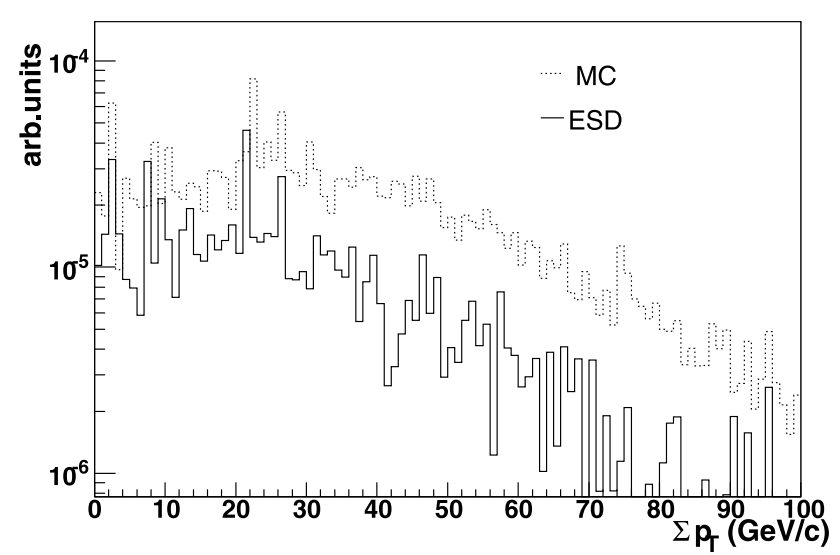

constructed by ALICE (solid line): $\gamma$-jet events (left) and jet-jet events $($ right $)$ in $p p$ collisions at $\sqrt{s}=14 \mathrm{TeV}$ generated in $\Delta \eta=0.26, \Delta \phi=$ $42^{\circ}$ and detected by two PHOS modules $\left(\Delta \eta=0.24, \Delta \phi=40^{\circ}\right)$

$20 \mathrm{GeV} / c$ ) are identified with the efficiencies and contamination as described in the previous sections. Then, from all charged tracks reconstructed in the central tracking barrel with $p_{\mathrm{T}_{\mathrm{h}}} \geq p_{\mathrm{T}_{\mathrm{h}}}^{\text {cut }}=2 \mathrm{GeV} / c$ the imbalance parameter $z_{\gamma-\mathrm{h}}$ is calculated. The relative azimuthal angle $\Delta \phi$ between the isolated photon and charged hadrons is strongly peaked at $\pi$ for $\gamma$-jet events (Fig. 5, left). In jet-jet events, the correlation shows a near and a far side peak, the later being shifted with respect to $\pi$. This is an artefact of the isolation cut which fails to identify mainly the decay photons emitted too far away from the jet axis so that the jet cone and the isolation-cut cone have only a small overlap (Fig. 5, right). To construct the imbalance distribution, we have selected events for which the photon-charged hadron satisfies the angular condition $\pi / 2<\Delta \phi<3 \pi / 2$. The resulting imbalance distribution (Fig. 6) for $p_{\mathrm{T}_{\gamma}}^{\text {cut }}=20 \mathrm{GeV} / c$ and $p_{\mathrm{T}_{\mathrm{h}}}^{\text {cut }}=2 \mathrm{GeV} / c$, normalised to the counting rate with two PHOS modules and corresponding to one standard year of data taking, indicate that the uncertainty in such kind of measurement is dominated by the systematic errors. The systematic errors could be reduced by selecting a sufficiently high photon energy, but measurements with higher $p_{\mathrm{T}_{\gamma}}^{\text {cut }}$ values are limited by the available counting rate.

The contribution to the imbalance function from the underlying $p p$ events was estimated by correlating the isolated photon with the charged hadrons emitted on the same side as the photon. We define our signal (S) as the opposite side correlated charged hadrons minus the same side correlated charged (B) and compare their $p_{\mathrm{T}}$ spectra (Fig. 7) to the one of the opposite side correlated charged hadrons $(\mathrm{S}+\mathrm{B})$. At $p_{\mathrm{T}}$ larger than $5 \mathrm{GeV} / c$, the contribution from the underlying event is small and stays constant, at the level of $2 \%$, over this $p_{\mathrm{T}}$ range. Therefore in the $z_{\gamma-\mathrm{h}}>0.2$ range, this contribution remains negligible. 


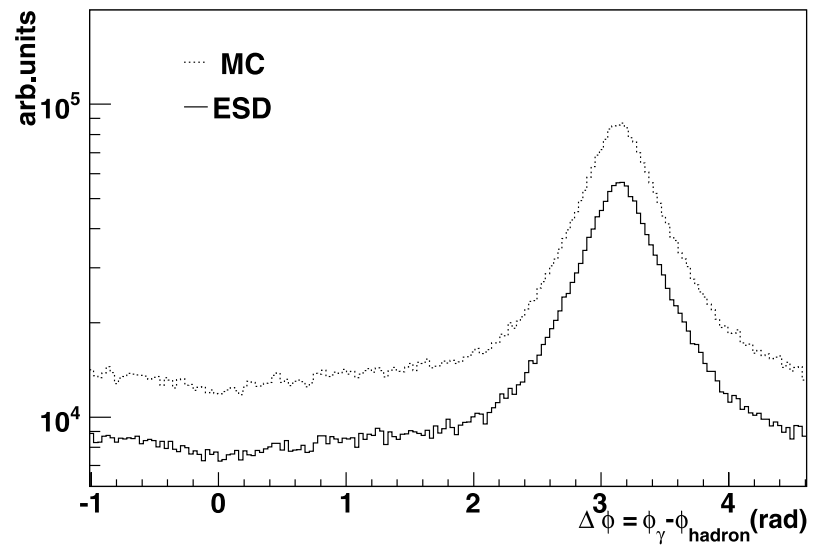

Fig. 5 Relative azimuthal angle distribution $\Delta \phi=\phi_{\gamma}-\phi_{\text {hadron }}$ in $p p$ collisions at $\sqrt{s}=14 \mathrm{TeV}$ for isolated photons with $p_{\mathrm{T}_{\gamma}}>20 \mathrm{GeV} / c$ : from Monte Carlo events (dashed line) and from events reconstructed

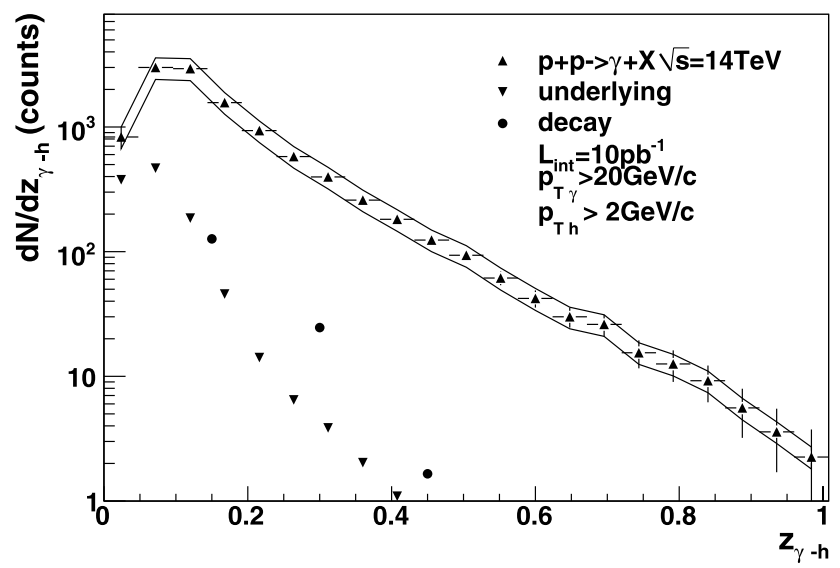

Fig. 6 Photon-charged hadrons imbalance distribution with $p_{\mathrm{T}_{\gamma}}>20 \mathrm{GeV} / c, p_{\mathrm{T}_{\mathrm{h}}}>2 \mathrm{GeV} / c$ calculated in $p p$ collisions at $\sqrt{s}=14 \mathrm{TeV}$ from PYTHIA generated Monte-Carlo data. The data points with error bars represents the total spectrum $(\gamma$-jet and jet-jet events) as it will be measured, the errors bars represent the statistical errors of the measurement and the area around the data points represents the systematic errors due to the jet-jet photons not properly identified. The contribution from jet-jet photons (closed circles) and from the underlying event (downwards triangles) are displayed as well

The imbalance distribution can, under these conditions be constructed for $z_{\gamma-\mathrm{h}} \gtrsim 0.2$ with an accuracy sufficient to measure medium effects of a magnitude of at least $10 \%$. Before validating the measurement to identify medium effects in heavy-ion collisions, it remains, however, to be investigated how the soft hadrons from the underlying heavy-ion event might distort the imbalance distribution and how much the systematic errors are increased because of the less efficient isolation selection.

To compare the imbalance distribution with the fragmentation function, the latter was constructed from the $\gamma$-jet events in the following way. Starting from the generated

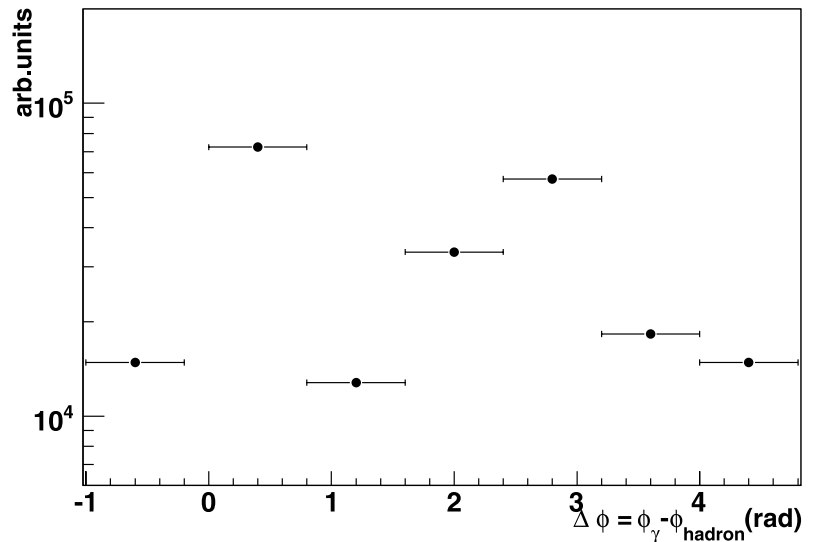

by ALICE (solid line) for $\gamma$-jet events (left) and jet-jet events (right). In jet-jet events the $\pi^{0}$ trigger is not necessarily the leading particle

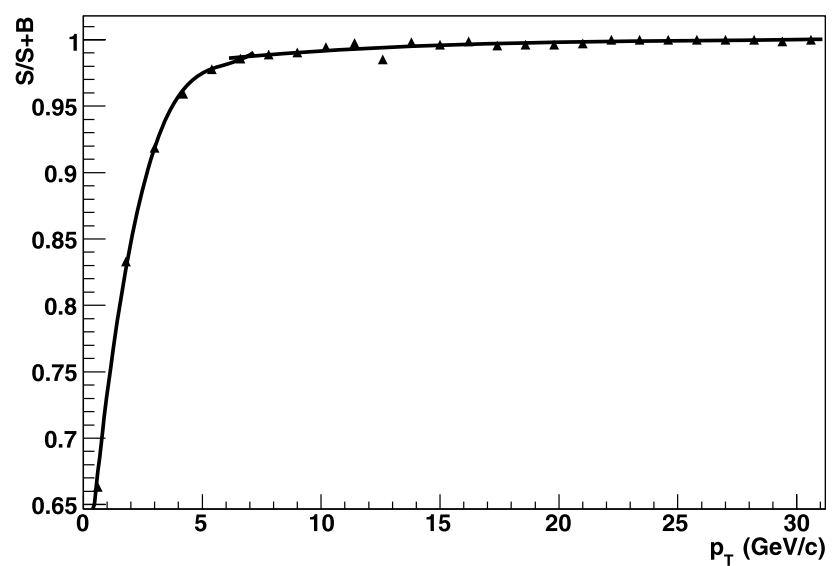

Fig. 7 The ratio of reconstructed signal hadrons from jet fragmentation to background hadrons from underlying events in $\gamma$-jet events. The solid line is a fit to the data points

events, the jet in each event was reconstructed $\left(E_{\mathrm{T}}^{\mathrm{jet}}, \eta_{0}, \phi_{0}\right)$ using the UA1 jet finder algorithm [21] implemented in the PYTHIA PYCELL cluster finding routine. The fragmentation function was then constructed from all particles $\left(p_{\mathrm{T}}\right.$, $\eta, \phi)$ within a cone of $R=\sqrt{\left(\eta-\eta_{0}\right)^{2}+\left(\phi-\phi_{0}\right)^{2}}=1$ around the jet direction as a function of $z=p_{\mathrm{T}} / E_{\mathrm{T}}^{\mathrm{jet}}$. Comparing the imbalance distribution for $p_{\mathrm{T}_{\gamma}}^{\text {cut }}=20 \mathrm{GeV} / c$, $p_{\mathrm{T}_{\mathrm{h}}}^{\text {cut }}=2 \mathrm{GeV} / c$ from Fig. 6 with the resulting fragmentation function scaled to the imbalance distribution in the range $z=0.1-0.8$ (Fig. 8), we observe that the two distributions are identical in the $z$ range between 0.12 and 0.65 and differ slightly at higher $z$ values by about $10 \%$ at $z=0.7$ and by about $40 \%$ at $z=0.9$ The difference below $z=0.12$ is a consequence of the kinematic cut applied in the construction of the imbalance distribution and the difference above $z=0.7$ is due to the overall transverse momentum resulting from the initial state $k_{\mathrm{T}}$ (set to $1 \mathrm{GeV} / c$ in our simu- 


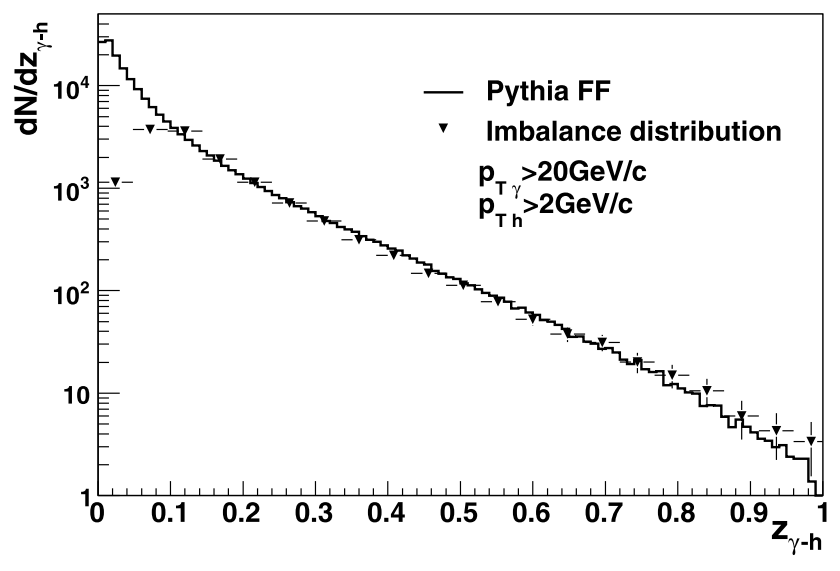

Fig. 8 Photon-charged correlation from Fig. 6 and fragmentation function unbiased by the detectors response and calculated from the PYTHIA generated Monte-Carlo $\gamma$-jet events. The data points represents the imbalance distribution and the histogram represents the fragmentation function normalised to the imbalance distribution in the range $z=0.1-0.8$

lation), the initial and final state radiation implemented in the PYTHIA generator. We have verified that these combined contributions modifies the imbalance distribution with respect to the fragmentation function similarly to a $k_{\mathrm{T}}$ of $5-6 \mathrm{GeV} / c$ without initial and final state radiation.

\section{Conclusion}

The feasibility to measure the imbalance distribution of prompt photons and charged hadrons has been evaluated in $p p$ collisions at LHC with the prospect to adapt the measurement to heavy-ion collisions. Because of the large particle density in heavy-ion collisions at LHC energies, jets with energies below $50 \mathrm{GeV}$ cannot be reconstructed with standard jet algorithms. This limit can be lowered exploiting photon tagging techniques. The imbalance distribution of prompt photons and charged hadrons provides the means to study the jet fragmentation function for these jets and identify medium effects such as those acting on the jet while traversing a color dense medium. Such a measurement should be rather sensitive to the medium and allow us to quantify the medium properties. We have shown with the present study that the measurement is feasible in $p p$ collisions, based on statistical and systematic errors arguments, with the ALICE experiment for jets of energy larger than $20 \mathrm{GeV}$. The photon spectrometer PHOS will detect and identify prompt photons using electromagnetic shower shape and isolation analysis. By selecting suitable kinematics conditions (prompt photons energy larger than $20 \mathrm{GeV}$ and correlated charged hadrons transverse momenta larger than $2 \mathrm{GeV} / \mathrm{c}$ ), the imbalance distribution can be measured with sufficient counting rate, the systematic errors, due to improperly identified $\pi^{0}$ decay photons and fragmentation photons and, at small values of $z$, to the underlying event contribution, remaining low enough for a meaningful measurement. The next step will be to study the feasibility of this measurement in heavy-ion collisions, in particular to study how the less efficient isolation criteria and the underlying event will modify the imbalance distribution under the same kinematic constraints and how well the signature of jet quenching is preserved.

Acknowledgements The authors gratefully acknowledge Dr. François Arleo for his enthusiastic discussions. Authors are supported partly by the CERN 2007 Summer Student Program (Yaxian Mao and Masato Sano), and partly by the NSFC (10575044 and key grant No. 10635020), the Key Project of Chinese Ministry of Education (306022 and IRT0624) and the Ministry of Science \& Technology of China (973: 2008CB317106) (Yaxian Mao and Daicui Zhou).

\section{References}

1. K. Adcox et al., Nucl. Phys. A 757, 184 (2005)

2. S. Adler et al., Phys. Rev. Lett. 91, 072301 (2003)

3. K.J. Eskola, H. Honkanen, C.A. Salgado, U.A. Wiedemann, Nucl. Phys. A 747, 511-529 (2005)

4. F. Karsch, E. Laermann, A. Peikert, Phys. Lett. B 605, 579 (2001)

5. C.A. Salgado, U.A. Wiedemann, Phys. Rev. Lett. 93, 42301 (2004)

6. A. Morsch, Nucl. Phys. A 783, 427 (2007)

7. G. Conesa et al., Nucl. Instrum. Methods Nucl. Res. A 580, 1446 (2007)

8. ALICE Collaboration. J. Phys. G: Nucl. Part. Phys. 30, 1517 (2004)

9. F. Arleo, J. Phys. G: Nucl. Part. Phys. 34(8), S1037 (2007). hep-ph/0701207

10. F. Arleo, P. Aurenche, Z. Belghobsi, J.-P. Guillet, J. High Energy Phys. 11, 009 (2004)

11. F. Arleo, J. High Energy Phys. 09, 015 (2006)

12. PHOS Technical Design Report, CERN/LHCC 99-4. http://alice. web.cern.ch/Alice/TDR/

13. T. Sjostrand et al., hep-ph/0108264 (2001)

14. H.L. Lai et al., Phys. Rev. D 55, 1280-1296 (1997). hep-ph/ 9606399

15. R. Brun et al., CERN Data Handling Division DD/EE/84-1, 1985

16. http://pcaliweb02.cern.ch/Offline/AliRoot/Manual.html

17. http://root.cern.ch

18. ALICE Collaboration, J. Phys. G: Nucl. Part. Phys. 32, 1295-2040 (2006)

19. G. Conesa et al., ALICE-INT-2005-16

20. G. Conesa et al., Nucl. Instrum. Methods Nucl. Res. A 585, 28 (2008)

21. UA1 Collaboration, C. Albajar et al., Nucl. Phys. B 309, 405 (1988) 